\title{
Eruptive Melanocytic Nevi Secondary to Encorafenib for BRAF Mutant Metastatic Colorectal Cancer
}

\author{
ALBERTO MENEGUZZO, ANNALISA LAZZAROTTO and MAURO ALAIBAC \\ Unit of Dermatology, University of Padua, Padua, Italy
}

\begin{abstract}
A 59-year-old woman, undergoing treatment with encorafenib for metastatic BRAF mutated colorectal cancer, developed during the first two months of therapy multiple eruptive nevi and changes in pre-existing nevi. Development of eruptive nevi has increasingly been reported in association with medications, most frequently conventional immunosuppressants and biologics. Some drugs are associated with eruptive nevi through an indirect effect of their mechanism of action, whereas other drugs are directly implicated in melanocyte proliferation. In this regard, BRAF inhibitors have been demonstrated to activate the MAPK pathway, and to promote cellular proliferation and survival, therefore leading to the development of new melanocytic nevi and to an increase in the size and hyperpigmentation of pre-existing nevi. A dermatological assessment and follow-up should be recommended in all patients presenting with eruptive nevi, regardless of the pathogenesis, because a high number of acquired melanocytic nevi may represent an adjunctive risk factor for melanoma.
\end{abstract}

Currently, eruptive nevi (EN) are not precisely defined in the literature. This term describes the sudden onset of multiple melanocytic lesions, usually over weeks to a month, associated with severe blistering skin diseases, conditions leading to compromised immunity, and the administration of medications including immunosuppressants (1-3). In particular, EN associated with medications (ENAMs) can be categorised into three distinct types, according to the classification proposed by Benjiamin et al. (2): Type I) eruptive nevi associated with immunosuppressants; Type II) eruptive nevi associated with chemotherapeutics; Type III) eruptive nevi associated with direct melanocyte stimulators.

This article is freely accessible online.

Correspondence to: Mauro Alaibac, Unit of Dermatology, University of Padua, via Gallucci 4, 35128 Padua, Italy. Tel: +39 0498212901,e-mail: mauro.alaibac@unipd.it

Key Words: Eruptive nevi, encorafenib, BRAF inhibitors, adverse effect, melanocytic nevi.
The most studied mechanism of $\mathrm{EN}$ is certainly immunosuppression (type I ENAMs). Several cases of EN have been reported to be caused by both conventional and immunosuppressant drugs, such as azathioprine, capecitabine, tocilizumab (anti-IL6), adalimumab (anti-TNF $\alpha$ ), rituximab (anti-CD20) (2) and natalizumab (anti-VLA4) (2, 4-8). It has been proposed that the modified immune surveillance in the skin may allow melanocyte growth factors to induce melanocyte proliferation in predisposed individuals $(9,10)$. Another hypothesis states that these melanocytic lesions develop as a result of a benign metastatic process, involving dissemination of an altered immature melanocytic progenitor cell (11). Although the pathogenesis is not understood, the development of new pigmented lesions in type I ENAMs seems to be linked to an indirect side effect of immunosuppression, and not to a direct effect of immunosuppressant drugs. It has been shown that some medications such as corticotrophin (12) and synthetic alpha melanocyte-stimulating hormone analogues (13) directly stimulate the development of EN (type III ENAMs). They are responsible for an increase in the circulating levels of melanocyte-stimulating hormone (MSH), which leads to diffuse hyperpigmentation through increased melanin production, and also to focal melanocyte proliferation, as observed in Addison's disease (14). A direct stimulation of melanocyte proliferation seems to be mediated by the effects of some chemotherapeutics (type II ENAMs); in particular, BRAF inhibitors (BRAFi), such as vemurafenib and encorafenib.

BRAF is a 766-amino acid, serine/threonine-specific protein kinase. Mutations in this gene seem to cause cancers by affecting cell division and differentiation. More than 30 mutations of the BRAF gene have been identified in association with human cancers with p.V600E being the most common mutation $(15,16)$. The presence of the $\mathrm{p}$. V600E mutation in $B R A F$ is considered a poor prognostic factor, as well as a potential biomarker of the lack of response to EGFR directed therapy in KRAS wild type colorectal cancers. Like melanoma, p.V600E in $B R A F$ is the most common mutation seen in the RAF family of proteins in colorectal cancers, but, unlike melanoma, the response of this cancer to anti-BRAF chemotherapeutics is limited. A possible strategy to overcome this resistance may be 


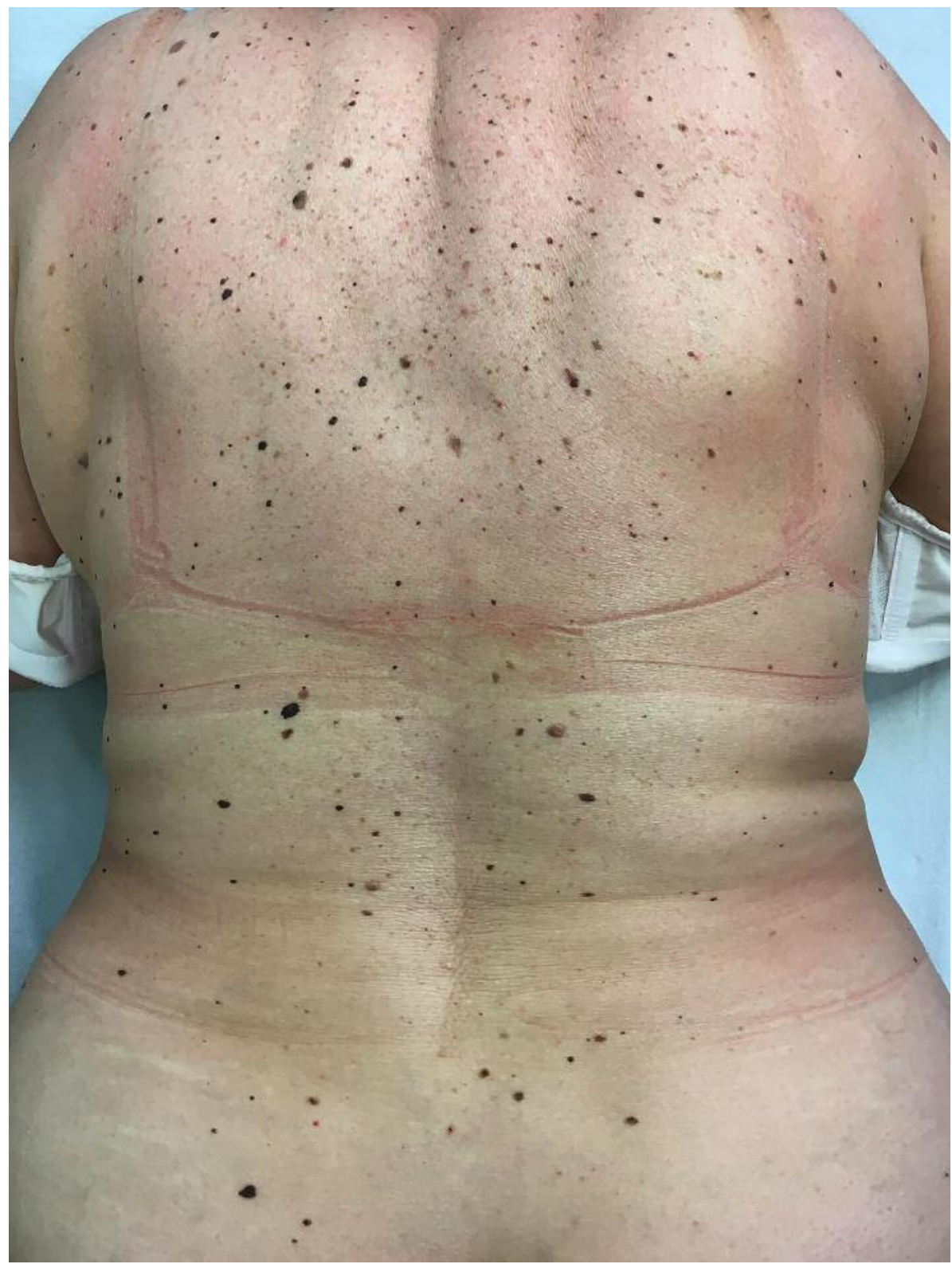

Figure 1. Eruptive melanocytic nevi of the back.

utilization of a combination therapy, with agents directed against EGFR and BRAF.

Encorafenib (LGX818) is a highly selective ATPcompetitive small molecule RAF kinase inhibitor, which suppresses the RAS-RAF-MEK-ERK pathway in tumor cells expressing the p.V600E $B R A F$ mutation. It is being investigated in phase III clinical trials for $B R A F$ mutant metastatic melanoma $(17,18)$ and in p.V600E BRAF mutant metastatic colorectal cancers (19), particularly in combination with MEK inhibitors. Patients undergoing BRAFi treatments without an association with an anti-MEK agent have been reported to develop new nevi or primary melanomas $(20,21)$.

In this article, we report the first case of eruptive nevi in a patient treated with encorafenib for p.V600E BRAF mutant colorectal cancer.

Case presentation. A 59-year-old woman was referred to our Dermatologic Unit for recent development of multiple eruptive new nevi; she also noted that the pre-existing nevi had changed both in size and in colour. 


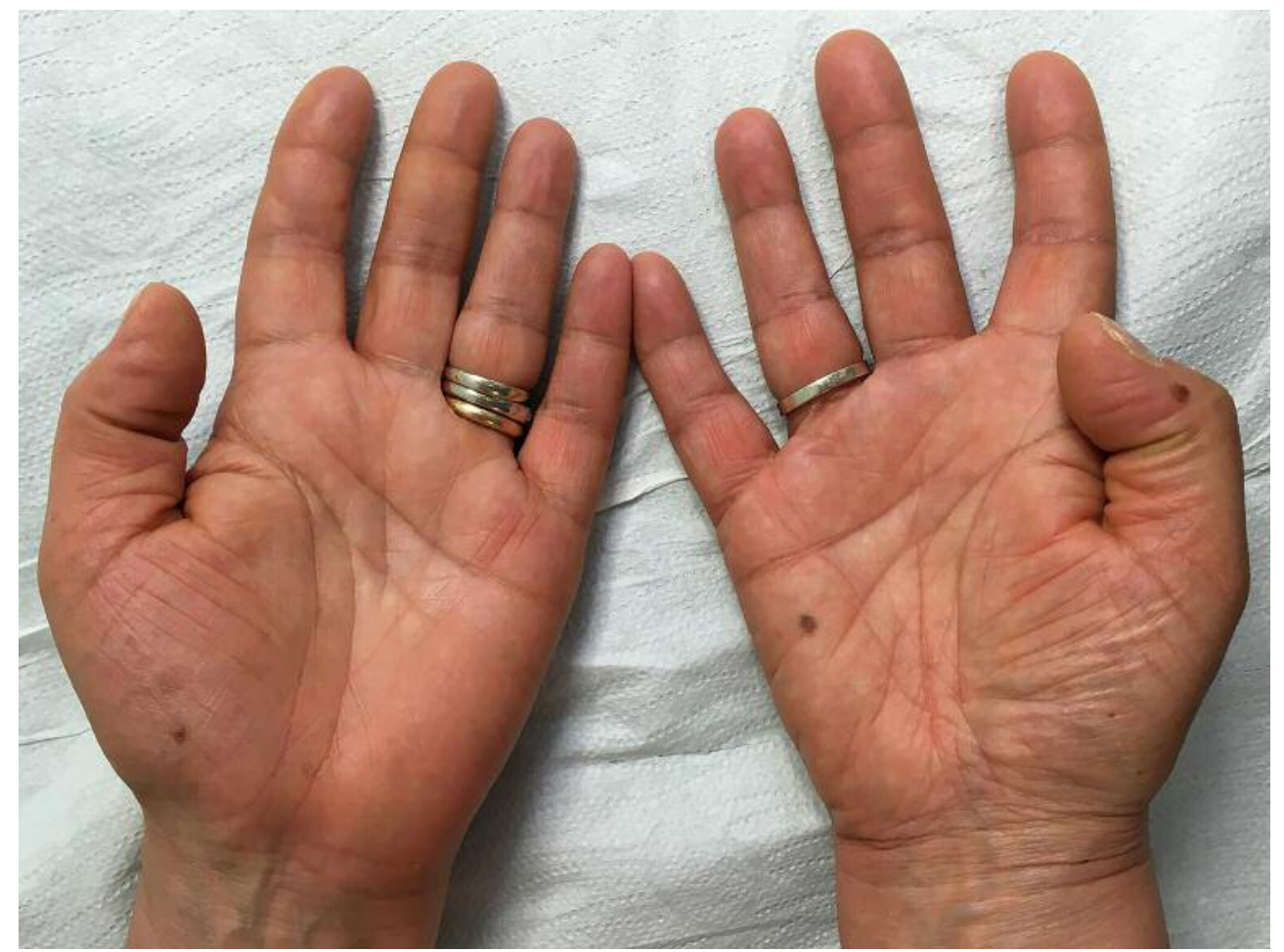

Figure 2. Eruptive melanocytic nevi of the palms.

The patient had been diagnosed approximately one year before our visit with advanced stage, $B R A F$ mutated colorectal cancer, and metastasis to abdominal lymph nodes and liver, and was not considered as a candidate for surgery. She had been treated with seven cycles of FOLFOXIRI protocol plus bevacizumab (a humanized monoclonal antibody that blocks angiogenesis by inhibiting vascular endothelial growth factor A) between February and September 2017. Few months later, this therapy was judged ineffective and was changed to encorafenib associated with cetuximab (a chimeric monoclonal antibody which binds to and inhibits EGFR) (22).

After two months in this therapeutic protocol, the patient begun to note the development of several new pigmented lesions throughout her body (Figure 1), including palms (Figure 2) and soles.

A complete dermatological assessment was performed, with full-body photography and dermoscopy, and the nevi were diagnosed clinically and dermoscopically as benign; consequently, the patient was followed up for 8-weeks. Two months later, the patient returned for follow-up dermatological assessment and was noted to have developed a few isolated new lesions, but the previous ones were stable.

\section{Discussion}

BRAF is a key enzyme in the MAPK signalling pathway (RAS-RAF-MEK-ERK), which regulates cellular proliferation, differentiation, survival and angiogenesis.

BRAF inhibitors' cutaneous toxicity is common, due to paradoxical activation of the MAPK pathway in wild type BRAF cells. It has been reported that treatment for 2-5 months resulted in different types of cutaneous toxicities, such as cutaneous squamous cell carcinoma, verrucal keratosis and plantar hyperkeratosis, Grover disease, hair follicle changes, panniculitis, photosensitivity, and eruptive nevi $(23,24)$.

Recent studies (25) have demonstrated that treatment with BRAFi induces proliferation of wild type (wt) BRAF cells in vivo, because of the activation of the RAS-RAF-MEKERK pathway, which promotes cell proliferation and survival. As a result, nevi increase in size and pigmentation, as we have described in our patient. Vice versa, the nevi that regressed during follow-up, were positive for the p.V600E $B R A F$ mutation.

Cutaneous toxicities are a well-known side effect of BRAFi; however, little is known about the specific cutaneous side-effect profile of LGX818. We presented a 
patient treated with LGX818 who developed a number of cutaneous toxicities after two months of treatment, most importantly, eruptive nevi. In our patient, new nevi developed, but changes in existing nevi also occurred. These included increase in the size and darkening of pigmentation of pre-existing nevi. Although BRAFi are quite specific for the $\mathrm{p} . \mathrm{V} 600 \mathrm{E}$ BRAF protein, in vitro, studies have demonstrated that, in vivo, BRAFi induce proliferation of wt BRAF cells (24). This suggests that in wt BRAF cells, the inhibitor promotes the formation of dimers between RAF molecules, facilitating the activation of MAPK pathway, which is responsible for cellular proliferation and survival. As a result, we observe changes in melanocytic nevi including increases in size, and hyperpigmentation.

\section{Conclusion}

Many questions remain to be answered regarding the clinical significance of EN, a fascinating phenomenon that occasionally occurs in association with several conditions, and after several treatments. In particular, some medications seem to be associated with eruptive nevi through an indirect effect of their mechanism of action, such as immunosuppression; instead, other drugs seem to be directly implicated in the proliferation and growth of melanocytes. The mechanisms involved are still partially unknown, but current research is focused on the BRAF gene and the effects that anti-BRAF chemotherapeutics have on the RAS-RAF-MEK-ERK pathway and its relations with melanocyte tropism. Further research is necessary before conclusions can be made.

Moreover, little is known about the risk of melanoma in patients who are administered drugs that can directly stimulate the development of new nevi. Therefore, it is important to instruct patients to report new skin lesions and to recommend regular dermatological assessment, in order to identify and remove any new nevus presenting clinical and/or dermoscopic atypia, or changing pigmented lesions of concern.

Regardless of the pathogenesis, a strict dermatological follow-up for the melanocytic lesions should be recommended in all patients presenting with $\mathrm{EN}$, because a high number of acquired melanocytic nevi represents itself a certain risk factor for melanoma skin cancer (25).

Considering this emerging association between anti-BRAF chemotherapeutics and EN, and the increasing use of this class of therapeutic agents, complete dermatological assessment and follow-up could reasonably be applied for all patients who begin treatment with a BRAFi.

\section{Conflicts of Interest}

The Authors have no conflicts of interest to declare regarding this study.

\section{Authors' Contributions}

$\mathrm{AM}, \mathrm{AL}$ and MA have made substantial contributions to acquisition of clinical data, have been involved in drafting the manuscript and have given final approval of the submitted manuscript.

\section{References}

1 Belloni-Fortina A, Piaserico S, Tonin E and Alaibac M: Melanoma and immunosuppression. Dermatology 218(1): 88, 2009. PMID: 18832814. DOI: 10.1159/000161125

2 Perry BM, Nguyen A, Desmond BL, Blattner CM, Thomas RS and Young RJ: Eruptive nevi associated with medications (ENAMs). J Am Acad Dermatol 75(5): 1045-1052, 2016. PMID: 27241804. DOI: $10.1016 /$ j.jaad 2016.04 .064

3 Zattra E, Fortina AB, Bordignon M, Piaserico S and Alaibac M: Immunosuppression and melanocyte proliferation. Melanoma Res 19(2): 63-68, 2009. PMID: 19194340. DOI: 10.1097/ CMR.0b013e328322fc20

4 Kravvas G and Kavanagh GM: Eruptive melanocytic naevi secondary to azathioprine: case report and review of the literature. Clin Exp Dermatol 43(1): 106-107, 2018. PMID: 28994130. DOI: $10.1111 /$ ced.13276

5 Calleja-Algarra A, Aragón-Miguel R, Velasco-Tamariz V, Andrés-Lencina JJ, Prieto Barrios $M$ and Maroñas Jiménez L: Eruptive nevi in a patient with metastatic breast cancer. Int $\mathrm{J}$ Dermatol 56(12): 1508-1509, 2017. PMID: 28960262. DOI: 10.1111/ijd.13717

6 Mansour Y, Lambert A, Tebacher-Alt M, Pasquali JL and Lipsker D: Eruptive nevi under tocilizumab: first case report and data analysis. J Eur Acad Dermatol Venereol 32(6): e253-e254, 2018. PMID: 29314272. DOI: $10.1111 / j d v .14784$

7 Moon IJ, Won CH, Lee MW, Choi JH and Chang SE: Eruptive benign melanocytic nevi formation following adalimumab therapy in a patient with Crohn's disease. Ann Dermatol 28(6): 777-779, 2016. PMID: 27904284. DOI: 10.5021/ad. 2016.28.6.777

8 Munguía-Calzada P, Vivanco B, Oliva-Nacarino P and SantosJuanes J: Melanoma, eruptive naevi and natalizumab: Causal relation or coincidence? Australas J Dermatol 58(4): 330-331, 2017. PMID: 28925067. DOI: 10.1111/ajd.12724

9 Woodhouse J and Maytin EV: Eruptive nevi of the palms and soles. J Am Acad Dermatol 52(5 Suppl 1): S96-S100, 2005. PMID: 15858520. DOI: 10.1016/j.jaad.2004.08.009

10 Bovenschen HJ, Tjioe M, Vermaat H, de Hoop D, Witteman BM and Janssens RW: Induction of eruptive benign melanocytic nevi by immune suppressive agents, including biologicals. $\mathrm{Br} \mathrm{J}$ Dermatol 154(5): 880-884, 2006. PMID: 16634890. DOI: 10.1111/j.1365-2133.2006.07189.x

11 Ross AL, Sanchez MI and Grichnik JM: Nevogenesis: A benign metastatic process? ISRN Dermatol 2011: 813513, 2011. PMID: 22363860. DOI: $10.5402 / 2011 / 813513$

12 Goldman L and Richfield DF: Effect of corticotrophin and cortisone on the development and progress of pigmented nevi. JAMA 143: 941-943, 1951. PMID: 14873600. DOI: 10.1001/ jama.1951.03670270031011

13 Cousen P, Colver G and Helbling I: Eruptive melanocytic nevi following melanotan injection. Br J Dermatol 161(3): 707-708, 2009. PMID: 19575725. DOI: 10.1111/j.1365-2133.2009.09362.x 
14 Ibsen $\mathrm{HH}$ and Clemmensen O: Eruptive nevi in Addison's disease. Arch Dermatol 126(9): 1239-1240, 1990. PMID: 2396848. DOI: 10.1001/archderm.126.9.1239

15 Karoulia Z, Gavathiotis E and Poulikakos PI: New perspectives for targeting RAF kinase in human cancer. Nat Rev Cancer 17(11): 676-691, 2017. PMID: 28984291. DOI: 10.1038/nrc.2017.79

16 Sanz-Garcia E, Argiles G, Elez E and Tabernero J: BRAF mutant colorectal cancer: prognosis, treatment, and new perspectives Ann Oncol 28(11): 2648-2657, 2017. PMID: 29045527. DOI: 10.1093/annonc/mdx401

17 Flaherty KT, Infante JR, Daud A, Gonzalez R, Kefford RF, Sosman J, Hamid O,Schuchter L, Cebon J, Ibrahim N, Kudchadkar R, Burris HA 3rd, Falchook G, Algazi A, Lewis K, Long GV, Puzanov I, Lebowitz P, Singh A, Little S, Sun P, Allred A, Ouellet D, Kim KB, Patel K and Weber J: Combined BRAF and MEK inhibition in melanoma with BRAF V600 mutations. N Engl J Med 367(18): 1694-703, 2012. PMID: 23020132. DOI: 10.1056/NEJMoa1210093

18 Koelblinger P, Thuerigen O and Dummer R: Development of encorafenib for BRAF-mutated advanced melanoma. Curr Opin Oncol 30(2): 125-133, 2018. PMID: 29356698. DOI: 10.1097/ CCO.0000000000000426

19 Sidaway P: Encorafenib - a new agent for advanced-stage disease. Nat Rev Clin Oncol 15(6): 344-345, 2018. PMID: 29651128. DOI: 10.1038/s41571-018-0019-x

20 Haenssle HA, Kraus SL, Brehmer F, Kretschmer L, Völker B and Asper H: Dynamic changes in nevi of a patient with melanoma treated with vemurafenib: importance of sequential dermoscopy. Arch Dermatol 148: 1183-1185, 2012. PMID: 22911096. DOI: 10.1001/archdermatol.2012.2649
21 Zimmer L, Hillen U, Livingstone E, Lacouture ME, Busam K and Carvajal RD: Atypical melanocytic proliferations and new primary melanomas in patients with advanced melanoma undergoing selective BRAF inhibition. J Clin Oncol 30: 23752383, 2012. PMID: 22614973. DOI: 10.1200/JCO.2011.41.1660

22 Van Geel RMJM, Tabernero J, Elez E, Bendell JC, Spreafico A and Schuler M: A phase Ib dose-escalation study of encorafenib and cetuximab with or without alpelisib in metastatic BRAFmutant colorectal cancer. Cancer Discov 7(6): 610-619, 2017. PMID: 28363909. DOI: 10.1158/2159-8290.CD-16-0795

23 Pascual LL, Muruzàbal RS, Gigli ML and Bayona JI: Cutaneous toxicity of a new BRAF inhibitor, LGX818 (encorafenib). Indian J Dermatol Venereol Leprol 83(1): 102-104, 2017. PMID: 27679406. DOI: 10.4103/0378-6323.191130

24 Anforth RM, Carlos GR, Scolyer RA, Chou S and FernandezPeñas P: Eruptive naevi in a patient treated with LGX818 for BRAF mutant metastatic melanoma. Melanoma Res 25(1): 91-94, 2015. PMID: 25380183. DOI: 10.1097/CMR.0000000000000127

25 Rastrelli M, Tropea S, Rossi CR and Alaibac M: Melanoma: epidemiology, risk factors, pathogenesis, diagnosis and classification. In Vivo 28(6): 1005-1011, 2014. PMID: 25398793.
Received August 29, 2019

Revised September 27, 2019

Accepted September 30, 2019 